\title{
3D Graphene Scaffolds for Skeletal Muscle Regeneration: Future Perspectives
}

\author{
Valentina Palmieri ${ }^{1,2 *}$, Francesca Sciandra ${ }^{3}$, Manuela Bozzi ${ }^{4}$, Marco De Spirito ${ }^{1,2}$ and \\ Massimiliano Papi ${ }^{1,2 *}$
}

${ }^{1}$ Dipartimento di Neuroscienze, Università Cattolica del Sacro Cuore, Rome, Italy, ${ }^{2}$ Fondazione Policlinico Universitario Agostino Gemelli IRCCS, Rome, Italy, ${ }^{3}$ Istituto di Scienze e Tecnologie Chimiche "Giulio Natta", (SCITEC)-CNR, SS Roma, Italy, ${ }^{4}$ Dipartimento di Scienze Biotecnologiche di Base, Cliniche Intensivologiche e Perioperatorie, Sezione di Biochimica e Biochimica Clinica, Università Cattolica del Sacro Cuore, Rome, Italy

\section{OPEN ACCESS}

Edited by:

Alfredo Maria Gravagnuolo, The University of Manchester,

United Kingdom

Reviewed by:

Renjie Chai,

Southeast University, China Hae-Won Kim,

Institute of Tissue Regeneration Engineering (ITREN), South Korea Miguel Angel Mateos Timoneda, Institute for Bioengineering of Catalonia (IBEC), Spain Jae Young Lee, Gwangju Institute of Science and Technology, South Korea

*Correspondence: Valentina Palmier valentina.palmieri@unicatt.it Massimiliano Papi massimiliano.papi@unicatt.it

Specialty section: This article was submitted to

Nanobiotechnology,

a section of the journal

Frontiers in Bioengineering and

Biotechnology

Received: 12 February 2020 Accepted: 07 April 2020 Published: 05 May 2020

Citation:

Palmieri V, Sciandra F, Bozzi M, De Spirito M, Papi M (2020) 3D Graphene Scaffolds for Skeletal Muscle Regeneration: Future

Perspectives.

Front. Bioeng. Biotechnol. 8:383. doi: 10.3389/fbioe.2020.00383
Although skeletal muscle can regenerate after injury, in chronic damages or in traumatic injuries its endogenous self-regeneration is impaired. Consequently, tissue engineering approaches are promising tools for improving skeletal muscle cells proliferation and engraftment. In the last decade, graphene and its derivates are being explored as novel biomaterials for scaffolds production for skeletal muscle repair. This review describes 3D graphene-based materials that are currently used to generate complex structures able not only to guide cell alignment and fusion but also to stimulate muscle contraction thanks to their electrical conductivity. Graphene is an allotrope of carbon that has indeed unique mechanical, electrical and surface properties and has been functionalized to interact with a wide range of synthetic and natural polymers resembling native musculoskeletal tissue. More importantly, graphene can stimulate stem cell differentiation and has been studied for cardiac, neuronal, bone, skin, adipose, and cartilage tissue regeneration. Here we recapitulate recent findings on 3D scaffolds for skeletal muscle repairing and give some hints for future research in multifunctional graphene implants.

Keywords: graphene, scaffold, skeletal muscle, tissue engineering, implants

\section{INTRODUCTION}

The discovery of methods to generate and genetically manipulate stem cells, the advances in biofabrication technologies including 3D bioprinting and the innovations biomimetic biomaterials are the three pillars of modern tissue engineering (Khademhosseini and Langer, 2016). The bidimensional flake of carbon, graphene, represents undoubtedly the revolutionary material of the last decade. Graphene has a hexagonal lattice structure of sp2 hybridized carbon atoms and is extremely thin $(<0.5 \mathrm{~nm})$, electrically and thermally conductive, mechanically resistant and light absorptive (Dreyer et al., 2014; Trusovas et al., 2016). Its derivative, the graphene oxide (GO) has oxygen functional groups decorating carbon plane and has largely been exploited in medicine due to its water dispersibility. Indeed pristine graphene is highly hydrophobic and tends to precipitate in biological media (Huang et al., 2020). GO has been functionalized with polymers and biomolecules and is also the precursor of rGO, the reduced form obtained by different chemical, hydrothermal, electrochemical procedures and similar to pristine graphene, but with defects and holes on the carbon skeleton (Dreyer et al., 2014; Palmieri et al., 2019a,b). Graphene-based materials (GBM) have been studied for several applications in biomedicine due to their unique interactions with 
proteins and molecules: from the first studies, it was evident that the surface area and chemistry of GBM allowed high adsorption of proteins that can mediate interactions with cells, bacteria and therapeutic compounds that can be delivered by graphene nanoflakes (Palmieri et al., 2018; Di Santo et al., 2019; Papi et al., 2019). Several methods to produce $3 \mathrm{D}$ graphene scaffolds have been designed, from hydrogels to electrospun graphene fibers and 3D printed GBM scaffolds (Zhang et al., 2018; Choe et al., 2019a; Li et al., 2019; Palmieri et al., 2020).

Graphene-based materials have been largely 3D printed or bioprinted (Palmieri et al., 2020). Though studies on 3D-printed GBM for cardiac muscles have been conducted (Choe et al., 2019a; Li et al., 2019), there is a lack of production of 3D printed GBM scaffolds for skeletal muscle regeneration. In this minireview, we want to define a primer to boost research on $3 \mathrm{D}$ printed implants for skeletal muscles based on GBM (Zhang et al., 2018). We foresee that graphene multi-functional scaffolds will represent the future of myo-regeneration based on 3D scaffolds.

\section{REPAIRING SKELETAL MUSCLE}

Skeletal muscle tissue is composed of multinucleated contractile muscle cells, the myofibers (Figure 1A). Parallel-aligned myofibers are bundled together to form fascicles and multiple fascicles are held by connective tissue to form mature muscle. Skeletal muscle can efficiently repair itself in response to injury (Tedesco et al., 2010). Muscle regeneration is a highly coordinated process that requires the recruitment of a pool of undifferentiated cells, called satellite cells. Satellite cells normally lie in a quiescence state beneath the basal lamina of muscle fibers but upon muscle injury are induced to proliferate, fuse and differentiate into multinucleated fibers leading to the complete regeneration of the injured muscle (Schultz and McCormick, 1994). Immediately after a muscle injury, proteolytic enzymes, cytokines and growth factors are released creating a local microenvironment that stimulates the migration of inflammatory cells and fibroblasts at the site of injury. A new temporary extracellular matrix (ECM) is produced stabilizing the tissue and acting as a scaffold to direct the migration of the satellite cells in the injured site (Charge and Rudnicki, 2004). Satellite cells are induced to differentiate in myoblasts that then fuse in mature myotubes. It is through this combination of ECM with muscle fibers that the injured muscle is repaired. This regeneration phase peaks at 2 weeks after injury and it also involves the generation of new blood vessels and nerves.

However, in chronic tissue damage, such as muscular dystrophies, or in acute severe muscle tissue loss due to traumatic injuries, skeletal muscle is unable to fully regenerate its structure leading to functional impairment and severe disability (Liu et al., 2018). Current cell therapies characterized by locally or systematically injection of stem cells or other myogenic/nonmyogenic cells are promising (Tedesco and Cossu, 2012) however, poor cell survival remains a challenge. GBM-scaffolds provide a structural synthetic framework that recreates the tridimensional microenviroment favorable for cell adhesion and proliferation (Jenkins and Little, 2019). In vitro analysis of these engineered muscles envisages morphological and functional evaluation of muscle maturation (Juhas et al., 2016), but the large-scale production of scaffolds induced the development of computational modeling systems for high-throughput testing (Torii et al., 2018).

\section{D GRAPHENE SCAFFOLDS AND MYOGENESIS}

For skeletal muscle regeneration, scaffolds need particular features to direct fusion of myocytes in multinucleated myotubes, stimulate vascularization and innervations. Moreover, materials have to degrade in a biocompatible way when the regenerated tissue is stable (Grasman et al., 2015; Torii et al., 2018). In Table 1, we summarize GBM 3D scaffolds produced and, in the following paragraphs, we analyze the advantages of using GBM scaffolds (Figure 1B).

\section{Graphene Composites}

Graphene composites for 3D scaffolds production are usually synthesized by combining a GBM and a synthetic or natural polymer. Natural biomaterials are usually biocompatible, easy to functionalize and primed for enzymatic degradation (Del Bakhshayesh et al., 2019). However, they are limited by batch-tobatch variability in chemical composition, and some components may be immunogenic. Synthetic polymers, on the other hand, are precisely mechanically and chemically controlled but degradation into byproducts and inflammatory responses might occur in response to this kind of material (Nakayama et al., 2019).

Graphene-based materials bring several advantages in skeletal muscle engineering. First, graphene derivatives can reinforce bare material mechanical properties and simulate muscle response to tension (Rogers and Liu, 2013; Hwang et al., 2019). GO films can mimic the adaptive behaviors of natural muscles including strengthening in response to strain (Dai et al., 2016) much more than other carbon nanomaterials. Natural polymers, like gelatin, collagen and chitosan, combined to GO can induce spontaneous differentiation of $\mathrm{C} 2 \mathrm{C} 12$ murine myoblasts cells (Shin et al., 2015; Lee et al., 2016; Patel et al., 2018). Graphene can also enhance materials biocompatibility. GO improves biocompatibility of microbeads of alginate for delivery of encapsulated C2C12 myoblasts (Ciriza et al., 2015) especially if embedded with a protective protein corona that mitigates in vivo foreign response (Ciriza et al., 2018). rGO-alginate hydrogels protect mesenchymal stem cells thanks to reactive oxygen species scavenging (Choe et al., 2019a). GO-alginate is bioprintable and bioconductive effects have been observed in many tissue engineering applications, paving the way for skeletal muscle reconstruction studies (Choe et al., 2019b).

As for neurons, electrical conductivity is essential for muscle cells that have indeed the ability to undergo depolarization and repolarization during cell contraction. The main advantage of electrical (and mechanical) stimulation in vitro for skeletal muscle cells is the mimicking of the physical simulation of stretch and electrical coupling in muscle. Ion channels on cell membranes generate an electrical potential within 


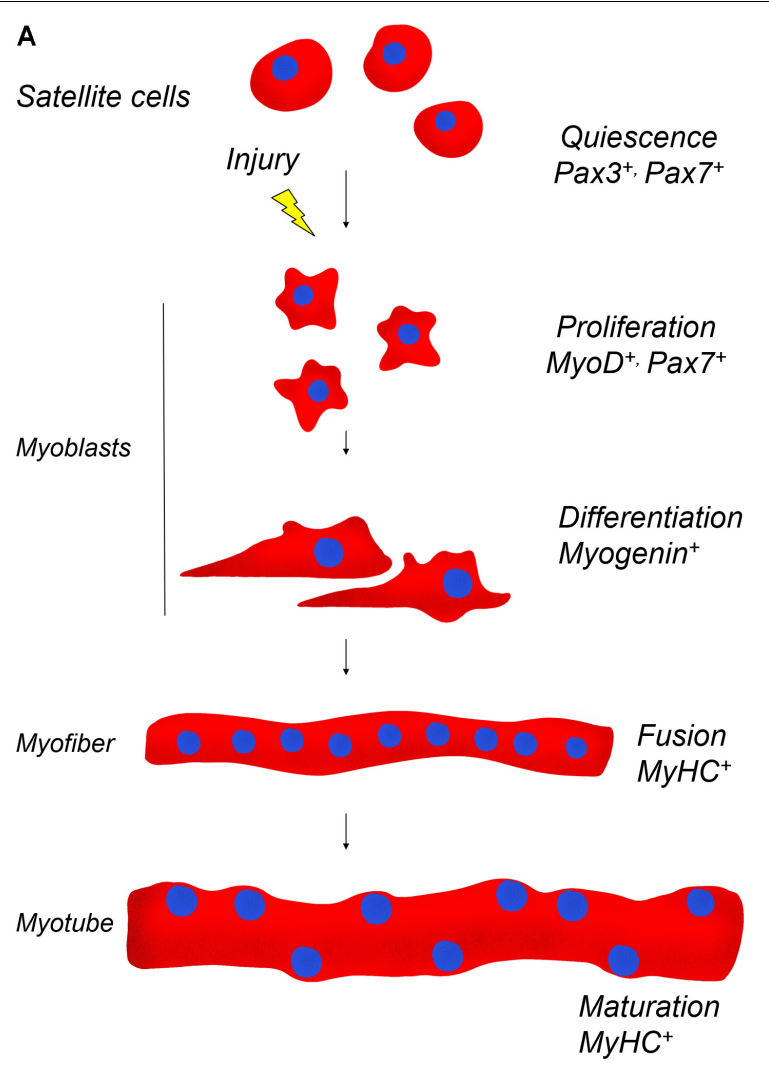

B Graphene-based materials (GBM)

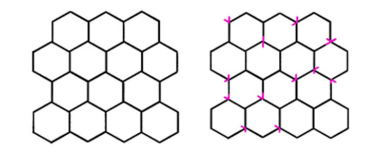

Graphene/Functionalized Graphene

\begin{tabular}{|ll|}
\hline \multicolumn{2}{|c|}{ 3D Scaffolds } \\
\hline - & Fibers \\
- & Foams \\
- & Hydrogels \\
\hline
\end{tabular}

$+$

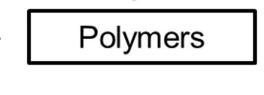

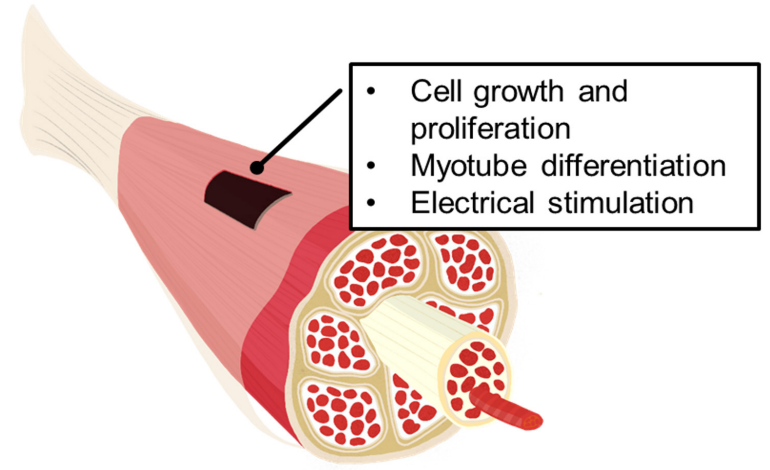

FIGURE 1 | (A) Skeletal muscle regeneration. Upon injury, quiescent muscle stem cells (satellite cells) undergo rapid proliferation, followed by differentiation into myoblasts, which fuse and mature to generate new muscle fibers. Muscle regeneration is characterized by the activation of transcription factors that are specific for each stage of the myofiber maturation process. In the figure, the most relevant modulators of myogenic lineage progression are indicated. (B) Illustration of GBM materials usage in skeletal muscle regeneration. GBM are obtained by combining graphene or graphene derivatives with natural or artificial polymers and a 3D scaffold is produced. GBM effects on muscle cells include induction of cell proliferation and differentiation. GBM are also conductive and can be electrically stimulated to favor muscle regeneration.

the membrane and application of electrical fields can alter the level of intracellular calcium content and cause signal transduction. This occurs in vivo for the regulation of muscular function by motor neurons and influences also cell attachment and proliferation. Electrical stimulation is known to cause myogenic differentiation and increase muscular forces (Nakayama et al., 2019). However, the exact mechanism that relates electrical stimulation and myogenic differentiation is still unknown though researchers hypothesized an involvement of calcineurin pathway mediated by the ion flow through cell membrane (Dong et al., 2019). rGO is known to have better electrical conductivity that can be further improved if other materials of the carbon family are anchored to its surface, like carbon nanotubes (Morimoto et al., 2016; Kim et al., 2017). A direct comparison of effects of rGO (obtained by hydrazine reduction) and $\mathrm{GO}$ substrates on $\mathrm{C} 2 \mathrm{C} 12$ cells was reported in an early study of $\mathrm{Ku}$ and Park (2013) with a better performance of GO in the induction of myogenic protein expression, multinucleate myotube formation, and expression of differentiation-specific genes (MyoD, myogenin, Troponin T, and $\mathrm{MHC}$ ). Nanotopography-mediated cell adhesion cannot explain differences between $\mathrm{GO}$ and $\mathrm{rGO}$, that have similar roughness.
However, the pro-differentiation effects of GO could be ascribed to its higher ability to adsorb serum proteins through its functional oxygen groups and ability to retain $3 \mathrm{D}$ conformation of proteins especially fibronectin, which is a mediator of muscle cell adhesion and differentiation (Ku and Park, 2013). Oppositely, rGO (obtained by ascorbic acid reduction) and polyacrylamide (PAAm) induce a significantly enhanced proliferation and myogenic differentiation compared with GO/PAAm and are electronically stimulable (Jo et al., 2017). These contrasting results could be explained by the mild reduction method employed by authors based on ascorbic acid, which possibly leaves a percentage of oxygen groups that might facilitate protein adsorption and consequently cell attachment. Alternatively, electrically conductive environments could per se facilitate electrical communication among muscle cells and result in the induction of increased myogenic differentiation besides external electrical stimulation. Poly (citric acid-octanediol-polyethylene glycol)-graphene (PCEG) composites have been produced and tested in vivo in rats as biodegradable and electrically conductive scaffolds. After subcutaneous implantation in rats, there was a lack of immunoreaction and a good capillary formation in the skeletal muscle lesion (Du et al., 2018). 
TABLE 1 | 3D graphene scaffolds for myoblasts growth and differentiation.

\begin{tabular}{|c|c|c|c|c|}
\hline & GBM composite & Cells & Effects & References \\
\hline \multirow[t]{5}{*}{ Self-assembling composites } & GO-Gelatin & $\mathrm{C} 2 \mathrm{C} 12$ & $\begin{array}{l}\text { Good cell growth and proliferation within the hydrogel } \\
\text { and spontaneous myogenic differentiation }\end{array}$ & Lee et al., 2016 \\
\hline & Graphene-CS-GG & $\mathrm{C} 2 \mathrm{C} 12$ & Efficient cell spreading, proliferation and differentiation & Patel et al., 2018 \\
\hline & GO-Alginate & $\mathrm{C} 2 \mathrm{C} 12$ & Increased viability of cells, for in vivo cell delivery & Ciriza et al., 2015 \\
\hline & GO-(PAAm); rGO-(PAAm) & $\mathrm{C} 2 \mathrm{C} 12$ & $\begin{array}{l}\text { Improved myoblast growth, myogenic differentiation } \\
\text { and electrical stimulation on rGO-(PAAm) compared to } \\
\text { GO-(PAAm), alignment along micropatterns }\end{array}$ & $\begin{array}{l}\text { Jo et al., 2017; } \\
\text { Park et al., } 2019\end{array}$ \\
\hline & rGO-PCEG & $\mathrm{C} 2 \mathrm{C} 12$ and in vivo & $\begin{array}{l}\text { Enhanced proliferation, differentiation and formation of } \\
\text { muscle fibers and blood vessels in vivo }\end{array}$ & Du et al., 2018 \\
\hline \multirow[t]{2}{*}{ Foams } & Graphene-laminin & $\mathrm{C} 2 \mathrm{C} 12$ & $\begin{array}{l}\text { Efficient differentiation into myotubes and contraction } \\
\text { upon electrical stimulus }\end{array}$ & $\begin{array}{l}\text { Krueger et al., } \\
2016\end{array}$ \\
\hline & GO-PU & $\mathrm{C} 2 \mathrm{C} 12$ & Promoted myogenic differentiation & Shin et al., $2018 \mathrm{a}$ \\
\hline \multirow[t]{5}{*}{ Electrospun fibers } & GO-RGD-PLGA & $\mathrm{C} 2 \mathrm{C} 12$ & $\begin{array}{l}\text { Enhanced adhesion, proliferation, myoblasts alignment } \\
\text { and fusion }\end{array}$ & Shin et al., 2018b \\
\hline & GO-PLGA-Collagen & $\mathrm{C} 2 \mathrm{C} 12$ & Enhanced attachment, proliferation and differentiation & Shin et al., 2015 \\
\hline & GO-PCL & C2C12; CB-hSkMCs & $\begin{array}{l}\text { Promoted myogenic differentiation and activation of } \\
\text { IGF signaling }\end{array}$ & $\begin{array}{l}\text { Chaudhuri et al., } \\
\text { 2014, } 2016\end{array}$ \\
\hline & Graphene-PCL & $\mathrm{C} 2 \mathrm{C} 12$ & $\begin{array}{l}\text { Promoted adhesion proliferation and differentiation in } \\
\text { growth media }\end{array}$ & Patel et al., 2016 \\
\hline & GO-PU & $\mathrm{C} 2 \mathrm{C} 12$ & $\begin{array}{l}\text { Enhanced initial adhesion and spreading, up-regulated } \\
\text { the myogenic mRNA levels and myosin heavy chain } \\
\text { expression. Mechanically stretchable fibers }\end{array}$ & Bin Jo et al., 2020 \\
\hline
\end{tabular}

CB-hSkMCs, human cord blood mesenchymal stem cells derived skeletal myoblast; CS, chitosan; GG, gellan gum; GO, graphene oxide; rGO, reduced graphene; PAAm, polyacrylamide; PCEG, Poly (citric acid-octanediol-polyethylene glycol); PCL, poly(e-caprolactone); PLGA, poly(lactic-co-glycolic acid); PU, polyurethane; RGD, Arg-Gly-Asp peptide.

\section{Graphene Foams}

Graphene foams 3D architectures consist of an interconnected lightweight continuous network of graphene sheets and has been used as an effective reinforcing agent in composites for biomedical and electronics (Idowu et al., 2018). Krueger et al. (2016) demonstrated that nickel/graphene foams can induce myotube formation if $\mathrm{C} 2 \mathrm{C} 12$ cells are seeded on it, especially if the foam is coated with laminin. Compared to $\mathrm{C} 2 \mathrm{C} 12$ cells cultured on planar graphene, the foams exhibited higher cell and myotube densities and have also been successfully used for electrical stimulation $( \pm 10 \mathrm{~V}, 50 \mathrm{~ms}$ duration, $1 \mathrm{~Hz})$ and induction of contraction of myotubes (Krueger et al., 2016).

Foams have been also produced by adding $\mathrm{GO}$ to polyurethane $(\mathrm{PU})$ and a spontaneous myogenic differentiation of myoblasts ascribed to the synergistic effects of GO and to the "community effect" was observed. This effect occurs when cells grown in the interconnected GO-PU foam pores, having an average size of $300 \mu \mathrm{m}$, have an augmented communication among the neighboring cells through cell-cell and cell-scaffold interactions (Shin et al., 2018a).

\section{GBM Topographies and Electrospun GBM Fibers}

Cell alignment is one of the most critical factors in skeletal muscle regeneration. Alignment can be obtained by both simulation of ECM topography and usage of fibrous elements within scaffolding materials (Grasman et al., 2015). Though the exact mechanism through which cells respond to topography is not well understood, it was shown that a period of $6 \mu \mathrm{m}$ is optimal for myoblast differentiation of myoblasts (Lam et al., 2006). While micropatterning has proven to be efficient in providing contact guidance to alignment, ECM proteins have nanoscale features that stimulate cytoskeletal-responsive pathways to enhance differentiation. Several bioengineering techniques aim to mimic the microenvironment topography features such as ripple and wrinkles that offer contacts for cell adhesion and can enhance stem cell differentiation (Grasman et al., 2015).

Graphene can modify micro and nano-features of 3D scaffolds. For example, the introduction of GO in hydrogel is used to increase the surface roughness (Zhou et al., 2018). Further, compressive strain-induced deformation of graphene substrates has been employed to form crumpled folds and cause the alignment and elongation of myoblasts (Kim et al., 2019). Nanotopographies can be added to surfaces of GBMcontaining scaffolds also through laser printing by exploiting light absorption properties of graphene (Papi et al., 2016; Palmieri et al., 2017). Park et al. (2019) used femtosecond laser ablation on hydrogels of GO and PAAm to evaluate the effects of stripes on myoblasts alignment. Micro-groove patterns $(20 \mu \mathrm{m}$ wide and $10 \mu \mathrm{m}$ deep) were designed on GO scaffolds at 20,50 or $80 \mu \mathrm{m}$ distance and then the scaffold was submerged in ascorbic acid to obtain rGO and enhance electrical properties of the samples (Park et al., 2019). rGO/PAAm with a $50 \mu \mathrm{m}$ pattern showed the best performance for differentiation and myotube alignment and, after the electrical stimulation of myoblasts, the differentiation was further enhanced. These implants were also biocompatible in vivo, i.e., didn't cause recruitment of inflammation cells (Park et al., 2019). 

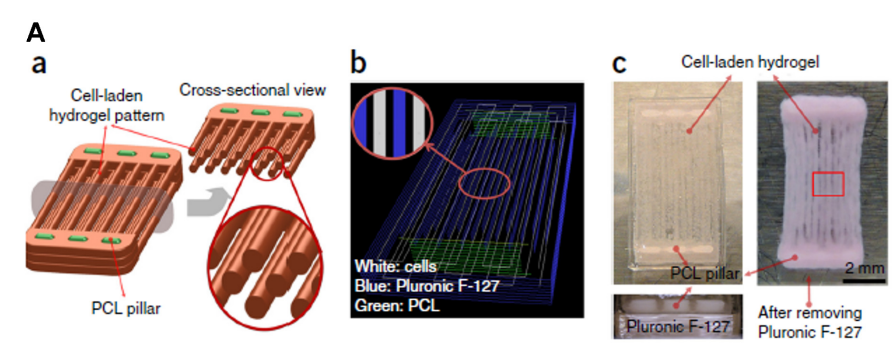

\section{B}
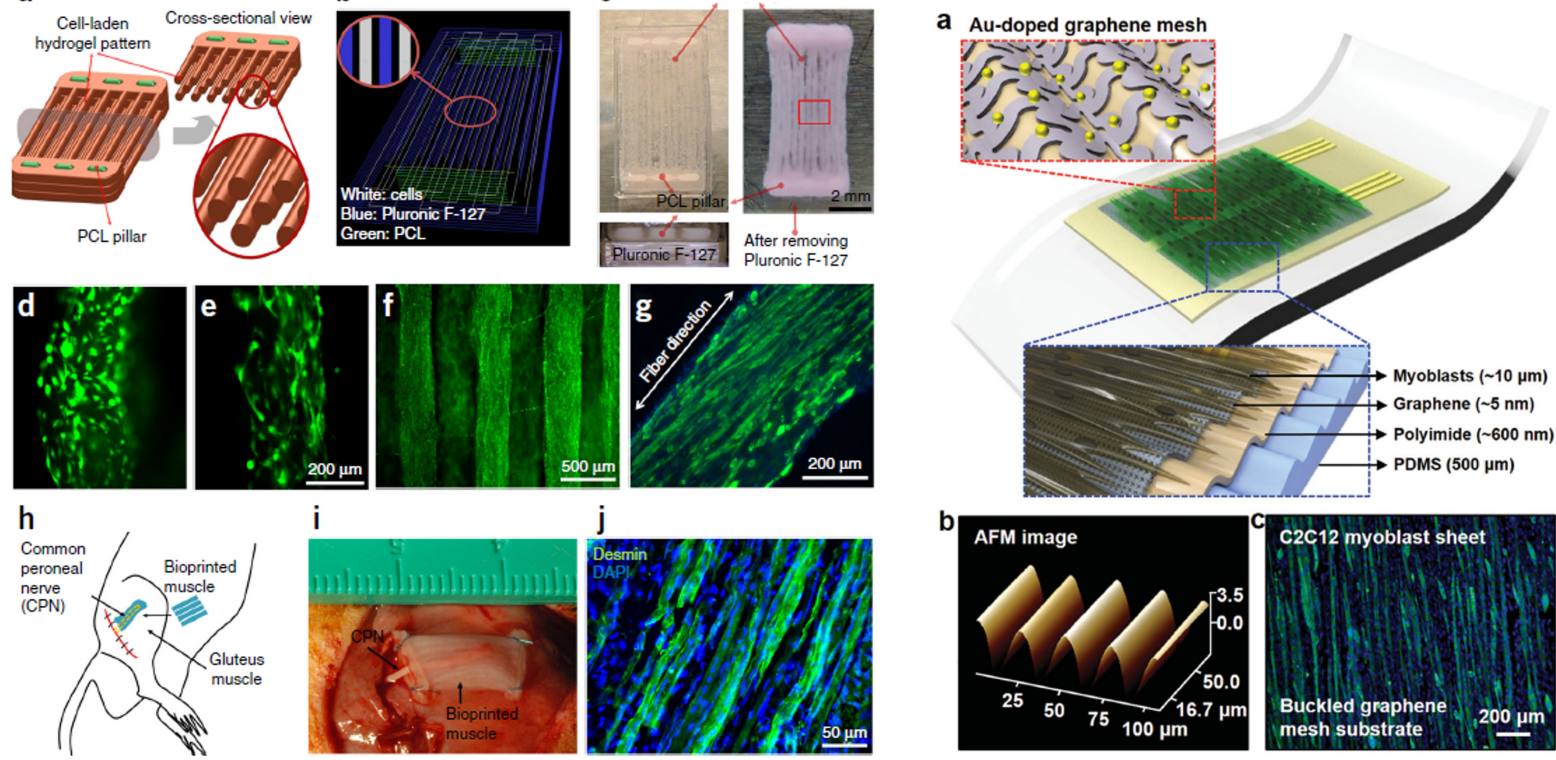

FIGURE 2 | (A) Bioprinted muscle. (a) Fiber bundle structure for muscle organization with PCL pillars (green) used to maintain the structure and to induce cell alignment. (b) Visualized motion program for 3D printing muscle construct. Lines of green, white and blue indicate the dispensing paths of PCL, cell-laden hydrogel and sacrificial material, respectively. (c) 3D patterning outcome of designed muscle organization before and after removing the sacrificial material. The PCL pillar structure is essential to stabilize the 3D printed muscle as visible from scaffolds without PCL pillar (d) and with PCL pillar (e). The cells with PCL pillar showed unidirectionally organized cellular morphologies (f). The live/dead staining of the encapsulated cells in the fiber structure indicates high cell viability (green: live; red: dead). (g) Immunofluorescent staining for myosin heavy chain of the 3D printed muscle organization after 7 days differentiation. (h) Schematic diagram of ectopic implantation of bioprinted muscle construct in vivo. (i,j) The bioprinted muscle construct subcutaneously implanted and the harvested implants after 2 weeks of implantation showed the presence of organized muscle fibers and innervating capability within the implanted construct, as confirmed by desmin muscle marker (j). Reproduced with permission from Kang et al. (2016). Copyright 2016 Nature America. (B) The architecture of the stretchable and transparent cell-sheet-graphene hybrid made of layers of C2C12 myoblasts, an ultrathin graphene mesh doped with Au, Au nanomembrane connective electrodes, polyimide and a PDMS substrate (a). Atomic force microscope image of graphene mesh (b) and C2C12 myoblasts (c) labeled for myosin heavy chain (green) and nuclei (blue). Reproduced with permission from Kim et al. (2016), Copyright Elsevier Ltd., 2019.

Electrospinning is a versatile technique to produce polymer nanofibers (diameter from 40 to $2000 \mathrm{~nm}$ ) forming 3D scaffolds and replicate aligned muscle architecture. Electrospinning is performed when the electric force of the mother liquid surface exceeds the surface tension and initiates an electric spark provoking the solution to be ejected from a syringe, and as jet flows the nanofiber is produced. Graphene gained significant interests for electrospinning researchers, for its high strength, flexibility, optical transparency, and conductivity (Javed et al., 2019; Parlayı $\mathrm{Cl}$ et al., 2019). Electrospinning fibers have been also used for muscle cells growth in several recent papers. GO-poly( $\varepsilon$-caprolactone (PCL) composites have been produced by Chaudhuri et al. (2016) to create a mesh with $\sim 85 \%$ porosity for $\mathrm{C} 2 \mathrm{C} 12$ cells growth. On GO-PCL, the myogenic proteins expression (Desmin and MyoD) and cell signaling were improved with a superior myogenic differentiation on these scaffolds, probably due to enhanced conductivity of the GO containing mesh. The same authors demonstrated that also human skeletal muscle cells derived from umbilical cord blood-derived mesenchymal stem cells, can form myotubes on GO-PCL (Chaudhuri et al., 2014). GO-PCL seems to have an effect on the insulin-like growth factor-1 pathway which is related to the myotube formation and maturation (Chaudhuri et al.,
2016). Also pristine graphene has been electrospun with PCL. Graphene-PCL had a decrease in impedance with the increase of graphene concentration (up to $2 \%$ ) and a good cytocompatibility besides being able to induce myogenic differentiation (Patel et al., 2016). The incorporation of GO in electrospun PU improved hydrophilicity, elasticity, and stress relaxation capacity (Bin Jo et al., 2020). PU/rGO composite nanofibrous electrospun scaffolds have been produced for cardiac tissue engineering. The rGO increased the electrical conductivity, Young's modulus and ultimate tensile strength and decreased the elongation at break of PU. On these scaffolds, troponin I gene expression was enhanced, especially when fibers were produced in aligned arrangement (Azizi et al., 2019). Electrospun fibers of poly(lacticco-glycolic acid, PLGA) and collagen with GO (GO-PLGA$\mathrm{Col})$ significantly improved the attachment and proliferation of the $\mathrm{C} 2 \mathrm{C} 12$ and stimulated the myogenic differentiation (Shin et al., 2015). Similarly, scaffolds of GO-PLGA enriched with RGD peptide (Arg-Gly-Asp) prompted myoblasts growth and differentiation (Shin et al., 2018b).

\section{D Printing in Skeletal Muscle Research}

Despite 3D printing has been successfully employed for the production of skin, adipose, bone and cardiac muscle 
(Li et al., 2019), limited research on skeletal muscle has been undertaken. 3D printed disks of polylactic acid are capable of myogenic differentiation induction thanks to cell proximity in printed channels (Rimington et al., 2017). PU bioink with muscle cells can be co-printed with fibroblastcontaining PCL to obtain differentiated stiffness and also stimulate tissue development and differentiation (Merceron et al., 2015; Choi et al., 2016). Interestingly both PU and PCL have been successfully combined to graphene derivatives in other studies and future research could be focused on muscletendon units improved on GBM scaffolds (Shin et al., 2018a; Palmieri et al., 2020).

Kang et al. (2016) created an integrated tissue-organ, printed and fabricated 3D muscle construct containing mouse myoblasts (Figure 2A). After 2 weeks, the retrieved muscle constructs showed well-organized muscle as well as nerve contacts and vascularization. Similarly, PEG/fibrinogen/alginate constructs generate a fully maturated muscle-like tissue (Costantini et al., 2017).

$3 \mathrm{D}$ printing has been employed to create artificial muscles that can be moved thanks to the photothermal properties of graphene that can be embedded in prosthesis and allow performing complex motions driven by laser light stimulation (Peele et al., 2015; Han et al., 2019; Helps et al., 2019; Zhou et al., 2019).

\section{DISCUSSION}

The studies reported in this mini-review demonstrated how scaffolds of GBM can induce myogenic differentiation. Additionally, GBM has been also used as a platform for neural cell growth, osteogenic differentiation, and chondrogenic differentiation, the major components of the musculoskeletal system. Besides being electrically activable, GBM have the intrinsic capability of (i) improve the viability of myoblasts (Ciriza et al., 2015) [and more generally of cells attached on surface, thanks to the adsorption of biomolecules and surface roughness (Zhou et al., 2018)] and (ii) induce myogenic differentiation probably facilitating the communication between cells (Jo et al., 2017). GBM have also been used in 2D films for muscle cell growth (Zhang et al., 2018), but we focused on $3 \mathrm{D}$ structures since these kinds of architectures recapitulate the native tissue inducing the expression of key myogenic modulators, parallel growth of muscle fibers and a correct organization of cytoskeleton and cell junctions increasing the community effect (Shin et al., 2018a; Naik et al., 2019). Interestingly, 3D printing of GBM has not been exploited for myogenesis, despite graphene inks and filaments are available. GBM can be useful for their electronic properties: GO and rGO have indeed been employed to create multifunctional stretchable and transparent devices implantable in vivo for electrostimulation and continuous monitoring of muscles (Figure 2Bb; Kim et al., 2016). Graphene layers have been transferred to 3D printed scaffolds of poly (methyl methacrylate) to improve the conductivity of the polymer (Kim et al., 2020) with possible applicability in muscle regeneration.
Besides the stimulation of myoblasts, in vivo implantation of graphene-based devices allows also neo-angiogenesis and this property should be exploited in the future also in skeletal muscle research (Kim et al., 2016; Du et al., 2018). GBM are indeed known to be capable of initiation of neurogenesis and neo-vascularization ( $\mathrm{Li}$ et al., 2019), paramount to prevent atrophy and tissue necrosis (Gilbert-Honick and Grayson, 2020). Traumatic musculoskeletal injuries are accompanied by loss of blood supply and denervation and GBM might fulfill multiple functions as long as the scaffold is engineered to work in compartments that selectively attach different kinds of cellular population, for example by bioprinting different porosities/concentrations as demonstrated by Holmes et al. for bone reconstruction (Holmes et al., 2016). Given the promising results of GBM on stem cells, it is surprising that there is no clinical translation of research findings. In vivo tests of GBM regeneration of muscle are limited to the implantation of PCEG containing rGO that didn't induce immune response (Du et al., 2018). However, this lack of immunogenicity cannot be generalized to all GBM materials: toxicity evaluation is made difficult by the infinite combinations of dose, shape, surface chemistry, exposure route and purity of graphenes used (Shareena et al., 2018). Future in vivo studies should foresee a strict application of guidelines to standardize the quality of toxicity evaluation (Reina et al., 2017). An ideal biomaterial, as well as matching native tissue compliance, should also degrade at a suitable rate to continue support during gradual reconstruction $(4 \sim 8$ weeks after muscular trauma (Winkler et al., 2011)). Biocompatibility and biodegradation are certainly the Achille's heel of GBM. GBM biodegradation is strictly dependent on the synthesis method, lateral size, and surface functionalization with polymers or proteins like albumin (Palmieri et al., 2019b; Syama and Mohanan, 2019). For this reason, it is difficult to generalize results on GBM degradability. However, it is ascertained that peroxidases or $\mathrm{H}_{2} \mathrm{O}_{2}$ alone can degrade graphene (Kotchey et al., 2011; Xing et al., 2014) and that the biodegradation of 3D scaffolds can be sped up by pretreatment with $\mathrm{O}_{2}$ plasma that increases hydrophilicity and number of defects (Loeblein et al., 2016). In conclusion, GBM promise to become the future exciting nanoplatforms for muscle engineering provided that nano-bio interactions and the toxic potential of GBM scaffold will be carefully evaluated.

\section{AUTHOR CONTRIBUTIONS}

All authors listed have made a substantial, direct and intellectual contribution to the work and approved it for publication.

\section{FUNDING}

The research leading to these results has received funding from AIRC under IG 2019 - ID. 23124 project - P.I. MP. Università Cattolica del Sacro Cuore contributed to the funding of this research project and its publication. 


\section{REFERENCES}

Azizi, M., Navidbakhsh, M., Hosseinzadeh, S., and Sajjadi, M. (2019). Cardiac cell differentiation of muscle satellite cells on aligned composite electrospun polyurethane with reduced graphene oxide. J. Polym. Res. 26:258.

Bin Jo, S., Erdenebileg, U., Dashnyam, K., Jin, G.-Z., Cha, J.-R., El-Fiqi, A., et al. (2020). Nano-graphene oxide/polyurethane nanofibers: mechanically flexible and myogenic stimulating matrix for skeletal tissue engineering. J. Tissue Eng. 11:2041731419900424. doi: 10.1177/2041731419900424

Charge, S. B. P., and Rudnicki, M. A. (2004). Cellular and molecular regulation of muscle regeneration. Physiol. Rev. 84, 209-238. doi: 10.1152/physrev.00019. 2003

Chaudhuri, B., Bhadra, D., Mondal, B., and Pramanik, K. (2014). Biocompatibility of electrospun graphene oxide-poly ( $\varepsilon$-caprolactone) fibrous scaffolds with human cord blood mesenchymal stem cells derived skeletal myoblast. Mater. Lett. 126, 109-112. doi: 10.1016/j.matlet.2014.04.008

Chaudhuri, B., Mondal, B., Kumar, S., and Sarkar, S. C. (2016). Myoblast differentiation and protein expression in electrospun graphene oxide (GO)poly ( $\varepsilon$-caprolactone, PCL) composite meshes. Mater. Lett. 182, 194-197. doi: 10.1016/j.matlet.2016.06.107

Choe, G., Kim, S.-W., Park, J., Park, J., Kim, S., Kim, Y. S., et al. (2019a). Anti-oxidant activity reinforced reduced graphene oxide/alginate microgels: mesenchymal stem cell encapsulation and regeneration of infarcted hearts. Biomaterials 225:119513. doi: 10.1016/j.biomaterials.2019.119513

Choe, G., Oh, S., Seok, J. M., Park, S. A., and Lee, J. Y. (2019b). Graphene oxide/alginate composites as novel bioinks for three-dimensional mesenchymal stem cell printing and bone regeneration applications. Nanoscale 11, 2327523285. doi: 10.1039/c9nr07643c

Choi, Y., Kim, T. G., Jeong, J., Yi, H., Park, J. W., Hwang, W., et al. (2016). 3D cell printing of functional skeletal muscle constructs using skeletal muscle-derived bioink. Adv. Healthc. Mater. 5, 2636-2645. doi: 10.1002/adhm.201600483

Ciriza, J., Del Burgo, L. S., Virumbrales-Munoz, M., Ochoa, I., Fernandez, L. J., Orive, G., et al. (2015). Graphene oxide increases the viability of C2C12 myoblasts microencapsulated in alginate. Int. J. Pharm. 493, 260-270. doi: 10.1016/j.ijpharm.2015.07.062

Ciriza, J., Saenz del Burgo, L., Gurruchaga, H., Borras, F. E., Franquesa, M., Orive, G., et al. (2018). Graphene oxide enhances alginate encapsulated cells viability and functionality while not affecting the foreign body response. Drug Deliv. 25, 1147-1160. doi: 10.1080/10717544.2018.1474966

Costantini, M., Testa, S., Mozetic, P., Barbetta, A., Fuoco, C., Fornetti, E., et al. (2017). Microfluidic-enhanced 3D bioprinting of aligned myoblastladen hydrogels leads to functionally organized myofibers in vitro and in vivo. Biomaterials 131, 98-110. doi: 10.1016/j.biomaterials.2017. 03.026

Dai, Z., Wang, Y., Liu, L., Liu, X., Tan, P., Xu, Z., et al. (2016). Hierarchical graphene-based films with dynamic self-stiffening for biomimetic artificial muscle. Adv. Funct. Mater. 26, 7003-7010. doi: 10.1002/adfm.201503917

Del Bakhshayesh, A. R., Asadi, N., Alihemmati, A., Nasrabadi, H. T., Montaseri, A., Davaran, S., et al. (2019). An overview of advanced biocompatible and biomimetic materials for creation of replacement structures in the musculoskeletal systems: focusing on cartilage tissue engineering. J. Biol. Eng. 13:85. doi: 10.1186/s13036-019-0209-9

Di Santo, R., Digiacomo, L., Palchetti, S., Palmieri, V., Perini, G., Pozzi, D., et al. (2019). Microfluidic manufacturing of surface-functionalized graphene oxide nanoflakes for gene delivery. Nanoscale 11, 2733-2741. doi: 10.1039/ c8nr09245a

Dong, R., Ma, P. X., and Guo, B. (2019). Conductive biomaterials for muscle tissue engineering. Biomaterials 229:119584. doi: 10.1016/j.biomaterials.2019.119584

Dreyer, D. R., Todd, A. D., and Bielawski, C. W. (2014). Harnessing the chemistry of graphene oxide. Chem. Soc. Rev. 43, 5288-5301. doi: 10.1039/c4cs 00060a

Du, Y., Ge, J., Li, Y., Ma, P. X., and Lei, B. (2018). Biomimetic elastomeric, conductive and biodegradable polycitrate-based nanocomposites for guiding myogenic differentiation and skeletal muscle regeneration. Biomaterials 157, 40-50. doi: 10.1016/j.biomaterials.2017.12.005

Gilbert-Honick, J., and Grayson, W. (2020). Vascularized and innervated skeletal muscle tissue engineering. Adv. Healthc. Mater. 9:1900626. doi: 10.1002/adhm. 201900626
Grasman, J. M., Zayas, M. J., Page, R. L., and Pins, G. D. (2015). Biomimetic scaffolds for regeneration of volumetric muscle loss in skeletal muscle injuries. Acta Biomater. 25, 2-15. doi: 10.1016/j.actbio.2015.07.038

Han, B., Zhang, Y., Zhu, L., Li, Y., Ma, Z., Liu, Y., et al. (2019). Plasmonic-assisted graphene oxide artificial muscles. Adv. Mater. 31:1806386. doi: 10.1002/adma. 201806386

Helps, T., Taghavi, M., and Rossiter, J. (2019). Thermoplastic electroactive gels for 3D-printable artificial muscles. Smart Mater. Struct. 28:85001.

Holmes, B., Bulusu, K., Plesniak, M., and Zhang, L. G. (2016). A synergistic approach to the design, fabrication and evaluation of $3 \mathrm{D}$ printed micro and nano featured scaffolds for vascularized bone tissue repair. Nanotechnology 27:64001. doi: 10.1088/0957-4484/27/6/064001

Huang, X.-M., Liu, L.-Z., Zhou, S., and Zhao, J.-J. (2020). Physical properties and device applications of graphene oxide. Front. Phys. 15:33301.

Hwang, T., Frank, Z., Neubauer, J., and Kim, K. J. (2019). High-performance polyvinyl chloride gel artificial muscle actuator with graphene oxide and plasticizer. Sci. Rep. 9:9658. doi: 10.1038/s41598-019-46147-2

Idowu, A., Boesl, B., and Agarwal, A. (2018). 3D graphene foam-reinforced polymer composites-A review. Carbon 135, 52-71. doi: 10.1016/j.carbon.2018. 04.024

Javed, K., Oolo, M., Savest, N., and Krumme, A. (2019). A review on graphene-based electrospun conductive nanofibers, supercapacitors, anodes, and cathodes for lithium-ion batteries. Crit. Rev. Solid State Mater. Sci. 44, 427-443. doi: 10.1080/10408436.2018.1492367

Jenkins, T. L., and Little, D. (2019). Synthetic scaffolds for musculoskeletal tissue engineering: cellular responses to fiber parameters. NPJ Regen. Med. 4:15. doi: 10.1038/s41536-019-0076-5

Jo, H., Sim, M., Kim, S., Yang, S., Yoo, Y., Park, J.-H., et al. (2017). Electrically conductive graphene/polyacrylamide hydrogels produced by mild chemical reduction for enhanced myoblast growth and differentiation. Acta Biomater. 48, 100-109. doi: 10.1016/j.actbio.2016.10.035

Juhas, M., Ye, J., and Bursac, N. (2016). Design, evaluation, and application of engineered skeletal muscle. Methods 99, 81-90. doi: 10.1016/j.ymeth.2015.10. 002

Kang, H.-W., Lee, S. J., Ko, I. K., Kengla, C., Yoo, J. J., and Atala, A. (2016). A 3D bioprinting system to produce human-scale tissue constructs with structural integrity. Nat. Biotechnol. 34, 312-319. doi: 10.1038/nbt.3413

Khademhosseini, A., and Langer, R. (2016). A decade of progress in tissue engineering. Nat. Protoc. 11, 1775-1781. doi: 10.1038/nprot.2016.123

Kim, J., Bae, S., Kotal, M., Stalbaum, T., Kim, K. J., and Oh, I. (2017). Soft but powerful artificial muscles based on 3D graphene-CNT-Ni heteronanostructures. Small 13:1701314. doi: 10.1002/smll.201701314

Kim, J., Leem, J., Kim, H. N., Kang, P., Choi, J., Haque, M. F., et al. (2019). Uniaxially crumpled graphene as a platform for guided myotube formation. Microsystems Nanoeng. 5:53. doi: 10.1038/s41378-019-0098-6

Kim, S. J., Cho, K. W., Cho, H. R., Wang, L., Park, S. Y., Lee, S. E., et al. (2016). Stretchable and transparent biointerface using cell-sheet-graphene hybrid for electrophysiology and therapy of skeletal muscle. Adv. Funct. Mater. 26, 32073217. doi: 10.1002/adfm.201504578

Kim, Y., Pagan-Diaz, G., Gapinske, L., Kim, Y., Suh, J., Solomon, E., et al. (2020). Integration of Graphene Electrodes with 3D Skeletal Muscle Tissue Models. Adv. Healthc. Mater. 4:1901137. doi: 10.1002/adhm.201901137

Kotchey, G. P., Allen, B. L., Vedala, H., Yanamala, N., Kapralov, A. A., Tyurina, Y. Y., et al. (2011). The enzymatic oxidation of graphene oxide. ACS Nano. 5, 2098-2108. doi: 10.1021/nn103265h

Krueger, E., Chang, A. N., Brown, D., Eixenberger, J., Brown, R., Rastegar, S., et al. (2016). Graphene foam as a three-dimensional platform for myotube growth. ACS Biomater. Sci. Eng. 2, 1234-1241. doi: 10.1021/acsbiomaterials.6b00139

$\mathrm{Ku}$, S. H., and Park, C. B. (2013). Myoblast differentiation on graphene oxide. Biomaterials 34, 2017-2023. doi: 10.1016/j.biomaterials.2012.11.052

Lam, M. T., Sim, S., Zhu, X., and Takayama, S. (2006). The effect of continuous wavy micropatterns on silicone substrates on the alignment of skeletal muscle myoblasts and myotubes. Biomaterials 27, 4340-4347. doi: 10.1016/j. biomaterials.2006.04.012

Lee, J. H., Lee, Y., Shin, Y. C., Kim, M. J., Park, J. H., Hong, S. W., et al. (2016). In situ forming gelatin/graphene oxide hydrogels for facilitated C2C12 myoblast differentiation. Appl. Spectrosc. Rev. 51, 527-539. doi: 10.1080/ 05704928.2016 .1165686 
Li, J., Liu, X., Crook, J. M., and Wallace, G. G. (2019). 3D graphene-containing structures for tissue engineering. Mater. Today Chem. 14:100199. doi: 10.1016/ j.mtchem.2019.100199

Liu, J., Saul, D., Böker, K. O., Ernst, J., Lehman, W., and Schilling, A. F. (2018). Current methods for skeletal muscle tissue repair and regeneration. Biomed Res. Int. 2018:1984879. doi: 10.1155/2018/1984879

Loeblein, M., Perry, G., Tsang, S. H., Xiao, W., Collard, D., Coquet, P., et al. (2016). Three-dimensional graphene: a biocompatible and biodegradable scaffold with enhanced oxygenation. Adv. Healthc. Mater. 5, 1177-1191. doi: 10.1002/adhm. 201501026

Merceron, T. K., Burt, M., Seol, Y.-J., Kang, H.-W., Lee, S. J., Yoo, J. J., et al. (2015). A $3 \mathrm{D}$ bioprinted complex structure for engineering the muscle-tendon unit. Biofabrication 7:35003. doi: 10.1088/1758-5090/7/3/035003

Morimoto, N., Kubo, T., and Nishina, Y. (2016). Tailoring the oxygen content of graphite and reduced graphene oxide for specific applications. Sci. Rep. 6:21715. doi: $10.1038 /$ srep 21715

Naik, A. R., Pernal, S., Lewis, K. T., Wu, Y., Wu, H., Carruthers, N. J., et al. (2019). Human skeletal muscle cells on engineered 3d platform express key growth and developmental proteins. ACS Biomater. Sci. Eng. 5, 970-976. doi: 10.1021/acsbiomaterials.8b01338

Nakayama, K. H., Shayan, M., and Huang, N. F. (2019). Engineering biomimetic materials for skeletal muscle repair and regeneration. Adv. Healthc. Mater. 8:1801168. doi: 10.1002/adhm.201801168

Palmieri, V., Barba, M., Di Pietro, L., Gentilini, S., Braidotti, M. C., Ciancico, C., et al. (2017). Reduction and shaping of graphene-oxide by laser-printing for controlled bone tissue regeneration and bacterial killing. 2D Mater. 5:15027. doi: 10.1088/2053-1583/aa9ca7

Palmieri, V., Bugli, F., Cacaci, M., Perini, G., De Maio, F., Delogu, G., et al. (2018). Graphene oxide coatings prevent Candida albicans biofilm formation with a controlled release of curcumin-loaded nanocomposites. Nanomedicine 13, 2867-2879. doi: 10.2217/nnm-2018-0183

Palmieri, V., Dalchiele, E. A., Perini, G., Motta, A., De Spirito, M., Zanoni, R., et al. (2019a). Biocompatible $\mathrm{N}$-acetyl cysteine reduces graphene oxide and persists at the surface as a green radical scavenger. Chem. Commun. 55, 4186-4189. doi: $10.1039 / \mathrm{c} 9 \mathrm{cc} 00429 \mathrm{~g}$

Palmieri, V., Lattanzi, W., Perini, G., Augello, A., Papi, M., and De Spirito, M. (2020). 3D-printed graphene for bone reconstruction*. 2D Mater. 7, doi: 10. 1088/1758-5090/aa6ed6

Palmieri, V., Perini, G., De Spirito, M., and Papi, M. (2019b). Graphene oxide touches blood: in vivo interactions of bio-coronated 2D materials. Nanoscale Horizons 4, 464-471. doi: 10.1039/c8nh00318a

Papi, M., Palmieri, V., Bugli, F., De Spirito, M., Sanguinetti, M., Ciancico, C., et al. (2016). Biomimetic antimicrobial cloak by graphene-oxide agar hydrogel. Sci. Rep. 6:12. doi: 10.1038/s41598-016-0010-7

Papi, M., Palmieri, V., Digiacomo, L., Giulimondi, F., Palchetti, S., Ciasca, G., et al. (2019). Converting the personalized biomolecular corona of graphene oxide nanoflakes into a high-throughput diagnostic test for early cancer detection. Nanoscale 11, 15339-15346. doi: 10.1039/c9nr01413f

Park, J., Choi, J. H., Kim, S., Jang, I., Jeong, S., and Lee, J. Y. (2019). Micropatterned conductive hydrogels as multifunctional muscle-mimicking biomaterials: Graphene-incorporated hydrogels directly patterned with femtosecond laser ablation. Acta Biomater. 97, 141-153. doi: 10.1016/j.actbio.2019.07.044

Parlay $l \mathrm{c} l$, \$̧, Avcl, A., and Pehlivan, E. (2019). Electrospinning of polymeric nanofiber (nylon 6,6/graphene oxide) for removal of Cr (VI): synthesis and adsorption studies. J. Anal. Sci. Technol. 10:13.

Patel, A., Xue, Y., Hartley, R., Sant, V., Eles, J. R., Cui, X. T., et al. (2018), Hierarchically aligned fibrous hydrogel films through microfluidic selfassembly of graphene and polysaccharides. Biotechnol. Bioeng. 115, 2654-2667. doi: 10.1002/bit.26801

Patel, A., Xue, Y., Mukundan, S., Rohan, L. C., Sant, V., Stolz, D. B., et al. (2016). Cell-instructive graphene-containing nanocomposites induce multinucleated myotube formation. Ann. Biomed. Eng. 44, 2036-2048. doi: 10.1007/s10439016-1586-6

Peele, B. N., Wallin, T. J., Zhao, H., and Shepherd, R. F. (2015). 3D printing antagonistic systems of artificial muscle using projection stereolithography. Bioinspir. Biomim. 10:55003. doi: 10.1088/1748-3190/10/5/055003

Reina, G., González-Domínguez, J. M., Criado, A., Vázquez, E., Bianco, A., and Prato, M. (2017). Promises, facts and challenges for graphene in biomedical applications. Chem. Soc. Rev. 46, 4400-4416. doi: 10.1039/c7cs00363c
Rimington, R. P., Capel, A. J., Christie, S. D. R., and Lewis, M. P. (2017). Biocompatible 3D printed polymers via fused deposition modelling direct $\mathrm{C}$ 2 C 12 cellular phenotype in vitro. Lab Chip. 17, 2982-2993. doi: 10.1039/ c7lc00577f

Rogers, G. W., and Liu, J. Z. (2013). Monolayer graphene oxide as a building block for artificial muscles. Appl. Phys. Lett. 102:21903.

Schultz, E., and McCormick, K. M. (1994). Skeletal Muscle Satellite cells, Rev. Physiol. Biochem. Pharmacol., 123, 213-257.

Shareena, T. P. D., McShan, D., Dasmahapatra, A. K., and Tchounwou, P. B. (2018). A review on graphene-based nanomaterials in biomedical applications and risks in environment and health. Nano-Micro Lett. 10:53. doi: 10.1007/s40820-0180206-4

Shin, Y. C., Kang, S. H., Lee, J. H., Kim, B., Hong, S. W., and Han, D.-W. (2018a). Three-dimensional graphene oxide-coated polyurethane foams beneficial to myogenesis. J. Biomater. Sci. Polym. Ed. 29, 762-774. doi: 10.1080/09205063. 2017.1348738

Shin, Y. C., Kim, C., Song, S.-J., Jun, S., Kim, C.-S., Hong, S. W., et al. (2018b). Ternary aligned nanofibers of RGD peptide-displaying M13 bacteriophage/PLGA/graphene oxide for facilitated myogenesis. Nanotheranostics 2:144. doi: 10.7150/ntno.22433

Shin, Y. C., Lee, J. H., Jin, L., Kim, M. J., Kim, Y.-J., Hyun, J. K., et al. (2015). Stimulated myoblast differentiation on graphene oxide-impregnated PLGAcollagen hybrid fibre matrices. J. Nanobiotechnol. 13:21. doi: 10.1186/s12951015-0081-9

Syama, S., and Mohanan, P. V. (2019). Comprehensive application of graphene: emphasis on biomedical concerns. Nano-Micro Lett. 11:6.

Tedesco, F. S., and Cossu, G. (2012). Stem cell therapies for muscle disorders. Curr. Opin. Neurol. 25, 597-603. doi: 10.1097/wco.0b013e32835 $7 \mathrm{f} 288$

Tedesco, F. S., Dellavalle, A., Diaz-Manera, J., Messina, G., and Cossu, G. (2010). Repairing skeletal muscle: regenerative potential of skeletal muscle stem cells. J. Clin. Invest. 120, 11-19. doi: 10.1172/jci40373

Torii, R., Velliou, R.-I., Hodgson, D., and Mudera, V. (2018). Modelling multi-scale cell-tissue interaction of tissue-engineered muscle constructs. J. Tissue Eng. 9:2041731418787141. doi: 10.1177/20417314187 87141

Trusovas, R., Račiukaitis, G., Niaura, G., Barkauskas, J., Valušis, G., and Pauliukaite, R. (2016). Recent Advances in Laser Utilization in the Chemical Modification of Graphene Oxide and Its Applications. Adv. Opt. Mater. 4, 37-65. doi: 10.1002/adom.201500469

Winkler, T., von Roth, P., Matziolis, G., Schumann, M. R., Hahn, S., Strube, P., et al. (2011). Time course of skeletal muscle regeneration after severe trauma: muscle function against the background of MRI and histological findings. Acta Orthop. 82, 102-111. doi: 10.3109/17453674.2010. 539498

Xing, W., Lalwani, G., Rusakova, I., and Sitharaman, B. (2014). Degradation of graphene by hydrogen peroxide. Part. Part. Syst. Charact. 31, 745-750.

Zhang, Z., Klausen, L. H., Chen, M., and Dong, M. (2018). Electroactive scaffolds for neurogenesis and myogenesis: graphene-based nanomaterials. Small 14:1801983. doi: 10.1002/smll.201801983

Zhou, J., Yang, X., Liu, W., Wang, C., Shen, Y., Zhang, F., et al. (2018). Injectable $\mathrm{OPF} /$ graphene oxide hydrogels provide mechanical support and enhance cell electrical signaling after implantation into myocardial infarct. Theranostics 8:3317. doi: 10.7150/thno.25504

Zhou, L., Gao, Q., Fu, J., Chen, Q., Zhu, J., Sun, Y., et al. (2019). Multimaterial 3D Printing of Highly Stretchable Silicone Elastomers. ACS Appl. Mater. Interfaces. 11, 23573-23583. doi: 10.1021/acsami.9b04873

Conflict of Interest: The authors declare that the research was conducted in the absence of any commercial or financial relationships that could be construed as a potential conflict of interest.

Copyright (c) 2020 Palmieri, Sciandra, Bozzi, De Spirito and Papi. This is an openaccess article distributed under the terms of the Creative Commons Attribution License (CC BY). The use, distribution or reproduction in other forums is permitted, provided the original author(s) and the copyright owner(s) are credited and that the original publication in this journal is cited, in accordance with accepted academic practice. No use, distribution or reproduction is permitted which does not comply with these terms. 